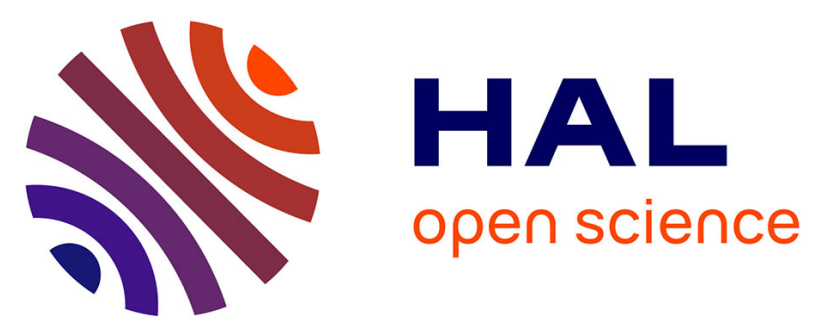

\title{
Étude du risque d'inondation d'un site industriel par des crues extrêmes: de l'évaluation des valeurs extrêmes aux incertitudes hydrologiques et hydrauliques
}

D. Alliau, J. de Saint Seine, M. Lang, Eric Sauquet, Benjamin Renard

\section{- To cite this version:}

D. Alliau, J. de Saint Seine, M. Lang, Eric Sauquet, Benjamin Renard. Étude du risque d'inondation d'un site industriel par des crues extrêmes: de l'évaluation des valeurs extrêmes aux incertitudes hydrologiques et hydrauliques. La Houille Blanche - Revue internationale de l'eau, 2015, 2015 (2), pp.67-74. 10.1051/lhb/20150021 . hal-01198719

\author{
HAL Id: hal-01198719 \\ https://hal.science/hal-01198719
}

Submitted on 15 Sep 2015

HAL is a multi-disciplinary open access archive for the deposit and dissemination of scientific research documents, whether they are published or not. The documents may come from teaching and research institutions in France or abroad, or from public or private research centers.
L'archive ouverte pluridisciplinaire HAL, est destinée au dépôt et à la diffusion de documents scientifiques de niveau recherche, publiés ou non, émanant des établissements d'enseignement et de recherche français ou étrangers, des laboratoires publics ou privés. 


\title{
Étude du risque d'inondation d'un site industriel par des crues extrêmes : de l'évaluation des valeurs extrêmes aux incertitudes hydrologiques et hydrauliques
}

\author{
Damien ALLIAU' ${ }^{1}$, Jacques DE SAINT SEINE ${ }^{1}$, Michel LANG², Eric SAUQUET², Benjamin RENARD ${ }^{2}$ \\ Compagnie Nationale du Rhône, 2 rue André Bonin,69316Lyon Cedex 04-d.alliau@cnr.tm.fr, j.desaintseine@cnr.tm.fr \\ 2.Irstea, 5 rue de la Doua, CS 70077,69626VVilleurbanne Cedex-michel.lang@irstea.fr,eric.sauquet@irstea.fr, benjamin.renard@irstea.fr
}

\begin{abstract}
RÉSUMÉ. - Dans le cadre de l'évaluation complémentaire menée suite aux événements japonais de mars 2011, l'exploitant d'un site industriel a souhaité pousser l'analyse des phénomènes naturels extrêmes d'inondation. Il a commandé une étude à CNR Ingénierie et Irstea sur l'évaluation du débit de la crue du Rhône pouvant atteindre le niveau de la plateforme industrielle avec son domaine d'incertitudes et la probabilité d'occurrence. L'étude a traité les points suivants : 1/ détermination de la relation hauteur-débit au droit de la plateforme et du débit de submersion ; $2 /$ calcul de la distribution prédictive des débits par ajustement d'une loi GEV sur les données disponibles à Saint-Etienne-des-Sorts (1845-2011), Viviers (1920-2011) et Beaucaire (1841-2011) ; 3/ propagation d'incertitudes des paramètres de la modélisation hydraulique à partir du couplage de l'outil statistique Prométhée de l'IRSN et du code de calcul hydraulique 1D Crue de la CNR. Des simulations à partir de 7000 tirs statistiques sur 9 paramètres ont permis de déterminer et de quantifier la dispersion des résultats selon la variation d'un ou plusieurs paramètres à la fois.
\end{abstract}

Mots-clés : Distribution prédictive, propagation d'incertitudes, méthode Monte-Carlo, Prométhée, Crue, hydrogramme.

\section{Flood study of industrial site by extreme flood risk: uncertainties with hydrologic and hydraulic extreme values}

\begin{abstract}
Under complementary assessment conducted after the Japanese events of 2011, operator of an industrial site wanted to study natural extreme flood. Operator commissioned a study by CNR and Irstea: discharge evaluation of increasing critical water level of the industrial platform in case of Rhone flooding, with associated uncertainties and probability. The study covered the following points: $1 /$ determination of the water level-discharge relationship near to the platform and the flooding flow; 2 / calculating the predictive distribution of floods by fitting a GEV law on data available at Saint-Etienne-des-Sorts (1845-2011), Viviers (1920-2011) and Beaucaire (1841-2011), 3 / propagation of uncertainties in hydraulic modeling from the coupling of the statistical tool Prométhée from IRSN and hydraulic calculation code 1D Crue from CNR. Simulations using 7000 statistical shots on 9 parameters can help us to identify and to quantify dispersion results according to the variation of one or more parameters at once.
\end{abstract}

Key-words: Predictive Distribution, propagation of uncertainties, Monte Carlo method, Prométhée, Crue, hydrograph.

Nota: les cotes altimétriques sont exprimées selon le système RGF-Orthométrique (Lallemand). L'écart positif moyen au système RGF-IGN69 est de $0.09 \mathrm{~m}$.

\section{INTRODUCTION}

Dans le cadre de l'évaluation complémentaire menée suite aux événements japonais de mars 2011, l'exploitant d'un site industriel a souhaité pousser l'analyse de phénomènes naturels extrêmes d'inondation, au-delà de ce qui était pris en compte jusque-là. En particulier, pour cette plateforme industrielle, ces évaluations ont porté sur le risque d'inondation externe résultant d'une crue du Rhône au-delà de la Cote Majorée de Sécurité.

L'exploitant s'est appuyé sur une étude hydraulique réalisée en 2005 par la Compagnie Nationale du Rhône (CNR Ingénierie), qui avait permis de définir la Cote Majorée de Sécurité avec un débit de $15675 \mathrm{~m}^{3} / \mathrm{s}$. Pour mémoire, le débit correspondant à la Cote Millénale Majorée est de $14930 \mathrm{~m} 3 / \mathrm{s}$. Cette étude a fait apparaître une marge de $16 \mathrm{~cm}$ entre la Cote Majorée de Sécurité et l'altitude de la plateforme industrielle (niveau zéro 38,70 m NGFO). Suite aux demandes de
l'Autorité compétente de compléter les évaluations déjà réalisées dans ce domaine, l'exploitant a souhaité que soit évalué le débit du Rhône assorti de ses incertitudes qui pourrait occasionner l'inondation de la plateforme industrielle.

\section{DEBIT DE SUBMERSION DE LA PLATEFORME INDUSTRIELLE}

L'exploitant a demandé une estimation des débits associés aux cotes 38,5 - 38,7 - 38,9 - 39 et 39,1 m NGFO au droit de la plateforme. Un modèle hydraulique avec le logiciel Crue a été construit par CNR Ingénierie, en actualisant les modèles existants à partir de levés topographiques récents. Le secteur modélisé couvre les biefs de Caderousse, d'Avignon et d'une partie de Vallabrègues sur une longueur de $32 \mathrm{~km}$, avec des casiers d'inondation susceptibles de se remplir en cas de submersion des endiguements CNR. 
Les endiguements dits "insubmersibles » de la CNR, dont le débit de dimensionnement dans le secteur est de $12500 \mathrm{~m}^{3} / \mathrm{s}$, n'ont pas été conçus pour résister à des déversements. Cependant leur rupture instantanée au premier déversement est peu probable. De ce fait, le comportement des endiguements de la CNR a fait l'objet de deux scénarios différenciés : le scénario 2 considère leur rupture dès les premiers déversements tandis que le scénario 1 n'envisage aucune rupture quelle que soit l'importance du déversement. Dans les deux cas, le lit majeur en rive gauche devient actif avec des capacités d'écrêtement variables selon le scénario.

Le site d'étude étant positionné à l'aval de la confluence du Rhône avec l'Ardèche (figure 1), dont les apports influencent très fortement les crues du Rhône, Irstea (anciennement Cemagref) a réalisé une étude hydrologique des formes d'hydrogrammes et proposé une typologie en trois classes, sur des bases méthodologiques inspirées de Sauquet et al. (2008) et Garçon et al. (2002), selon que la crue est : 1/ mixte (Rhône et Ardèche), 2/ issue de 1'Ardèche seule ou 3/ du Rhône seul. La situation la plus pénalisante est obtenue avec une crue de classe 3 (Rhône seul) : pour un débit de pointe donné, elle possède le plus fort volume de crue. Des hydrogrammes ont été élaborés par homothétie de forme afin d'alimenter les conditions aux limites des modèles numériques. La figure 2 suivante présente les niveaux déterminés par la modélisation hydraulique pour

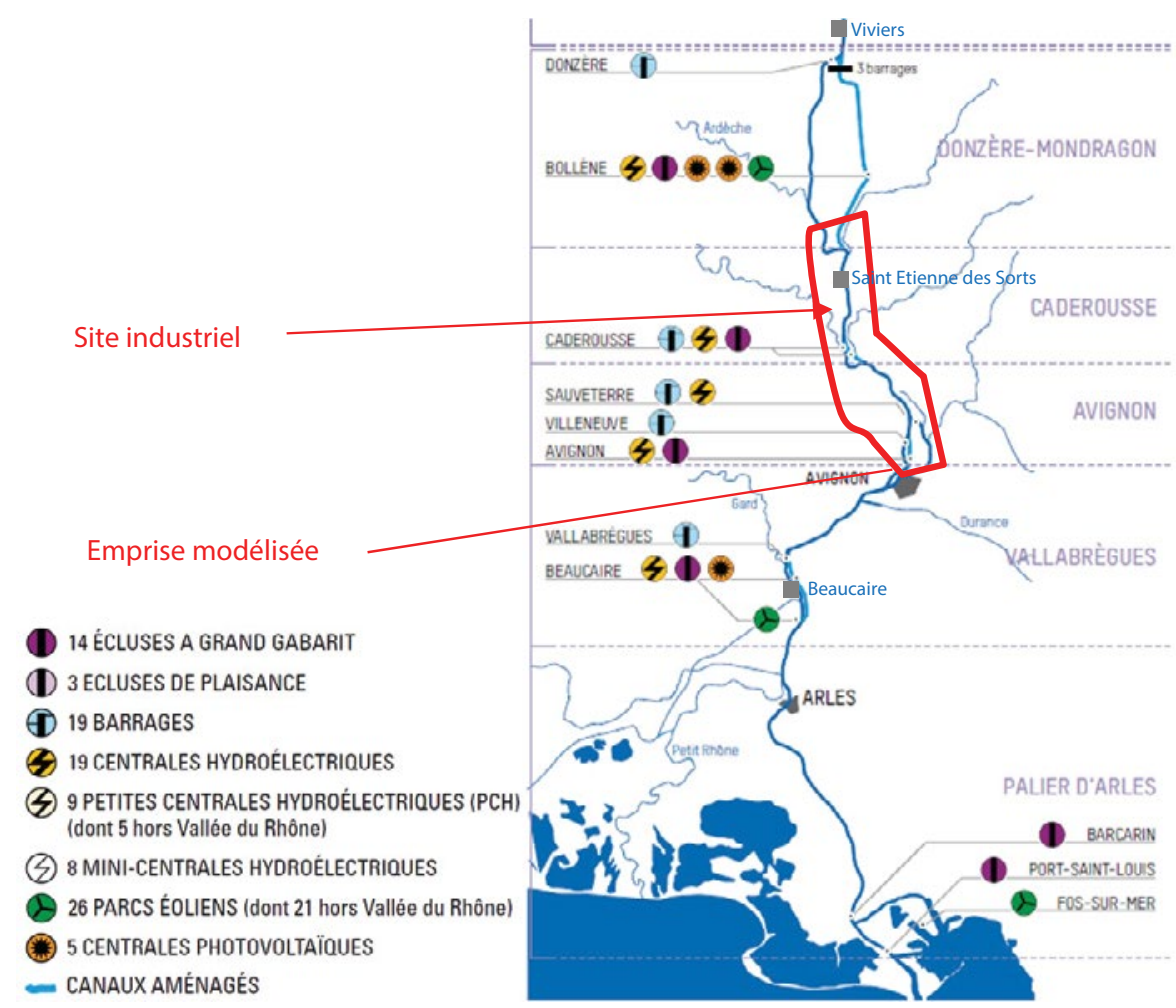

Figure 1 : Localisation du périmètre d'étude sur le Bas Rhône (source : cartographie des aménagements CNR, 2013).

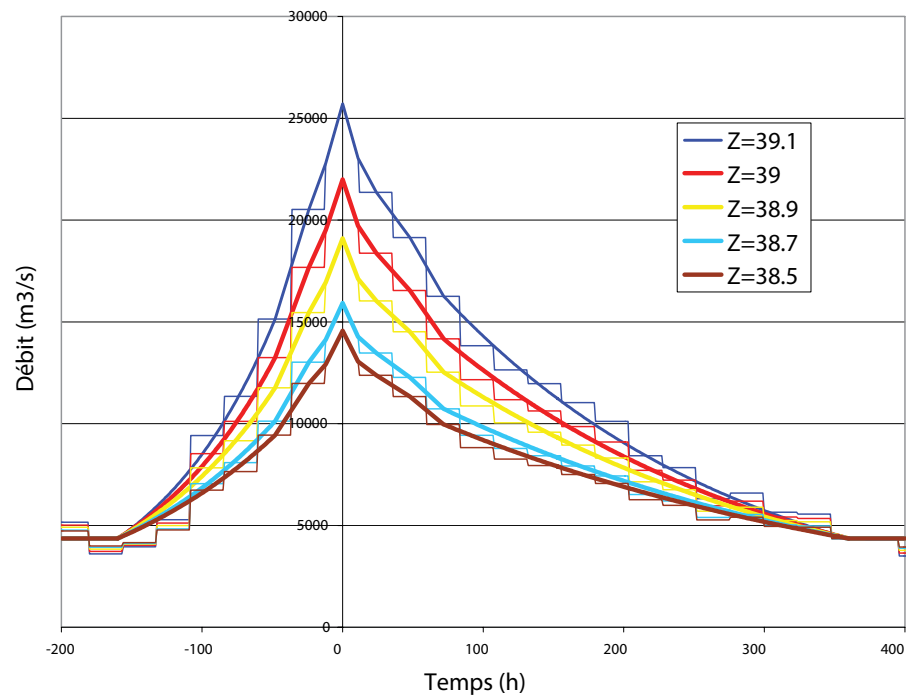

Figure 2 : Hydrogrammes de crue de classe 3 (Rhône seul) associés à différents niveaux de submersion au droit de la plateforme (scénario 1 sans rupture des endiguements $C N R$ ). 
différents hydrogrammes de classe 3 . Les hydrogrammes ont été construits à partir des informations disponibles en débit moyen journalier, puis lissés à pas de temps plus fin.

Les résultats du scénario 1 , sans rupture, des simulations hydrauliques montrent que le niveau zéro de la plateforme $(\mathrm{Z}=38,7 \mathrm{~m})$ est atteint à partir d'un débit de $15929 \mathrm{~m} / \mathrm{s}$. Les débits associés aux cotes 38,5 - 38,9-39 et $39,1 \mathrm{~m}$ NGFO sont respectivement de 14 565, 19100,22000 et $25711 \mathrm{~m}^{3} / \mathrm{s}$. La figure 2 illustre les différents hydrogrammes associés à ces débits de pointe au droit de la plateforme.

A dire d'expert, les cotes atteintes le long des endiguements (non conçus pour résister à la surverse) pour des débits dépassant $14565 \mathrm{~m}^{3} / \mathrm{s}$ devraient entraîner de façon quasi-certaine une rupture de ces derniers (lame d'eau supérieure à la dizaine de centimètres pendant plusieurs dizaines d'heures). Les écrêtements de débit consécutifs à l'activation $\mathrm{du}$ lit majeur rive gauche limitent alors la cote au droit de la plateforme à 38,5 m NGFO.

Dans la partie suivante, nous rechercherons les périodes de retour associées à ces différents débits, puis nous examinons la sensibilité de ces résultats dits «nominaux » vis-à-vis des incertitudes portées par l'ensemble des paramètres hydrauliques.

\section{DISTRIBUTION PRÉDICTIVE ET EVALUATION DES DEBITS EXTREMES}

Dans le cadre de cette étude, nous allons utiliser la loi généralisée des valeurs extrêmes (GEV) (Fisher et Tippett, 1928) :

$$
\begin{aligned}
F(x)=\operatorname{Prob}(X<x)= & \exp \left\{-\left[1-k \frac{x-x_{0}}{a}\right]^{1 / k}\right\} \text { loi } \operatorname{GEV}(k \neq 0) \\
& \exp \left\{-\exp \left(-\frac{x-x_{0}}{a}\right)\right\} \quad \text { loi } \operatorname{Gumbel}(k=0)
\end{aligned}
$$

On distingue trois cas de figure : loi de Weibull $(k>0)$, loi de Gumbel $(k=0)$, loi de Fréchet $(k<0)$. La loi de Weibull est bornée supérieurement par $x_{0}+a / k$. La loi de Gumbel est plus robuste à ajuster qu'une loi GEV, le paramètre de forme $k$ étant très sensible à l'échantillon utilisé, lorsque celui-ci ne comporte que quelques dizaines de valeurs. Nous avons considéré deux modes de calcul des quantiles de crue :

- l'estimation modale, en utilisant le meilleur ensemble de paramètres $\hat{\rho}$ fourni par la méthode d'estimation :

$$
\widehat{F}(x)=\int_{0}^{x} f(x \mid \hat{\rho}) d x
$$

- la distribution prédictive, qui prend en compte l'incertitude d'échantillonnage $f(\rho)$ sur les paramètres :

$$
F_{\text {pred }}(x)=\int_{0}^{x} \int_{\rho} f(x \mid \rho) f(\rho) d x d \rho
$$

La distribution prédictive comporte une intégration de la distribution des débits de crue suivant tous les jeux de paramètres possibles $\rho$, pondérés par leur probabilité respective $f(\rho)$. Elle fournit une estimation de quantiles, qui prend en compte le risque de se tromper sur l'estimation des paramètres de la distribution. En ce sens, elle offre un calcul plus élaboré, du point de vue des fondements théoriques, que la règle actuelle de calcul de cote de sûreté des installations (ASN, 2013), basée sur la borne supérieure de l'intervalle de confiance à $70 \%$ de la crue millénale, majorée de $15 \%$. Par ailleurs, aucune indication n'est donnée sur la loi à utiliser. Nous verrons dans la suite de l'étude que la largeur de l'intervalle de confiance varie assez nettement en fonction de la loi de probabilité utilisée (loi de Gumbel ou GEV) et de la taille de l'échantillon.

Nous présenterons dans un premier temps les résultats obtenus à partir des données récentes en débit journalier maximal annuel $Q J X$ de la station de Saint-Etienne-des-Sorts (1955-2007).

La figure 3 montre que baser uniquement les estimations de période de retour à partir de la série hydrométrique de Saint-Etienne-des-Sorts (1955-2007), la plus proche de la plateforme industrielle, conduit à de fortes incertitudes d'estimation sur le débit moyen journalier $Q J X(1000)$ de la crue millénale, qui vaut respectivement en valeur modale 10400 et $13500 \mathrm{~m}^{3} / \mathrm{s}$ avec une loi de Gumbel $(k=0)$ et une loi GEV $(k=-0.127)$. Les incertitudes sont certainement sous-estimées avec une loi de Gumbel et surtout, le choix d'une loi de Gumbel ne s'avère pas justifié (cf. valeur nulle du coefficient de forme de la loi GEV juste à la limite de l'intervalle de confiance à $90 \%$, IC90). Par ailleurs, l'estimation du paramètre de forme de la loi GEV s'avère très imprécise avec une série courte de 52 années et conduit à un intervalle de confiance très large. Le calcul en mode prédictif pour la loi GEV donne des valeurs très élevées qui ne semblent pas réalistes compte-tenu de la présence de zones d'expansion dans le lit majeur.

Afin de réduire ces incertitudes d'estimation, nous avons étendu les données analysées en exploitant un rapport ancien de la CNR daté de mars 1973, qui contient un graphique de Gumbel avec le report de la distribution empirique des valeurs instantanées maximales annuelles QIX à Saint-Etienne-des-Sorts sur la période 1845-1963. Les données de débit ont été déduites graphiquement, avec 101 valeurs lues pour 119 années. La mention sur le document "par analogie avec la station d'Avignon" indique que ces valeurs ont dû être reconstituées à partir de la série des cotes maximales à Avignon disponible depuis 1845. Nous avons ensuite concaténé ces 101 valeurs de débit de pointe avec celles disponibles sur la période 1964-2011. Les deux séries ont des propriétés statistiques similaires en terme de moyenne et d'écart-type (cf. non rejet de l'hypothèse d'homogénéité, avec un seuil d'erreur de $1 \%$ ).

Nous avons comparé les résultats obtenus à SaintEtienne-des-Sorts (série concaténée 1845-2011) avec ceux obtenus à partir des longues séries disponibles en amont et en aval, à Viviers (1920-2012) et à Beaucaire (1841-2012). La figure 4a montre un comportement de type Fréchet pour la distribution des crues ajustée à Saint-Etienne-des-Sorts, à l'inverse de celui des deux autres distributions. Etant donné le caractère incertain de la série concaténée des débits de pointe de Saint-Etienne-des-Sorts, avec sur la période 1845-1963 des valeurs lues sur un graphique de Gumbel sans connaissance précise du mode d'obtention des données, et sur la période récente 1964-2011 trois sous-périodes et des modes d'exploitation différents (1950-1974 : mesure de cote et conversion en débit à l'aide d'une courbe de tarage ; 1975-1995 : débits reconstitués au droit du barrage CNR; 1996-2011 : station ultrasons de Chusclan), nous privilégions le comportement de type Weibull obtenu aux stations hydrométriques de Viviers et Beaucaire. 

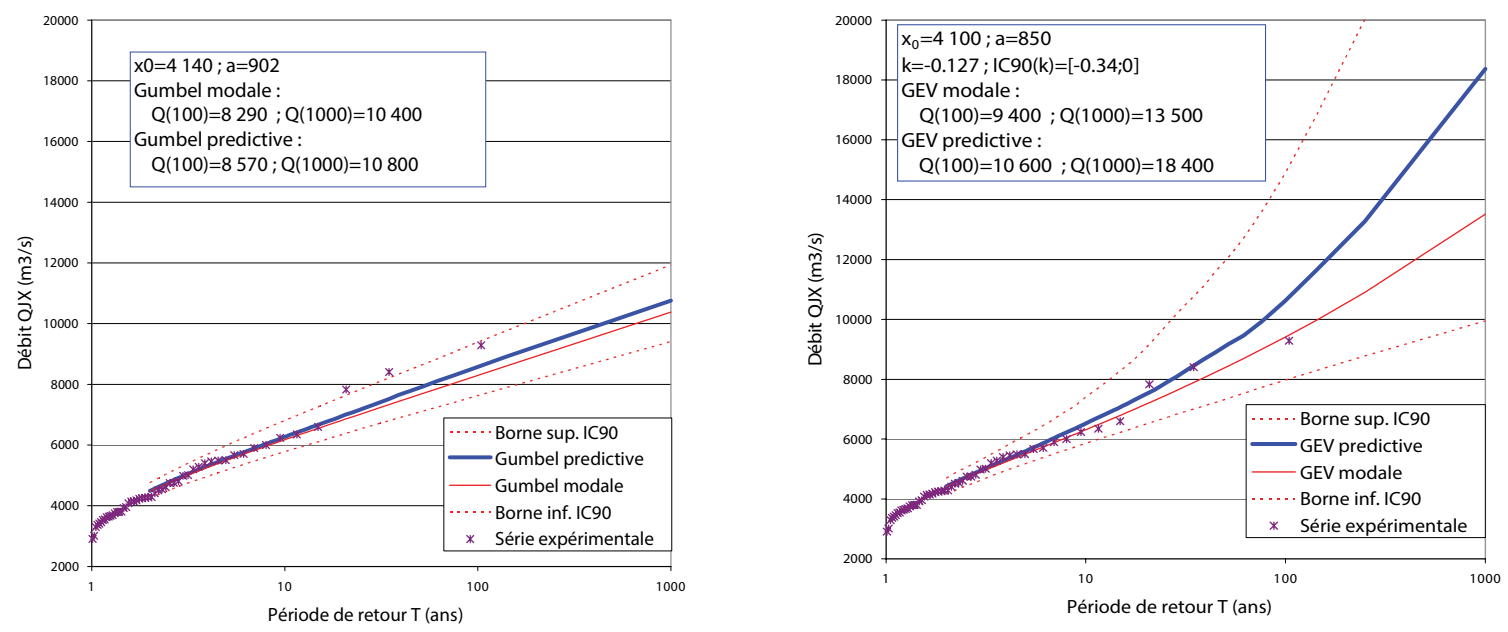

Figure 3 : Distribution des débits QJX à Saint-Etienne-des-Sorts (1955-2007) : a/ loi de Gumbel ; b/ loi GEV.
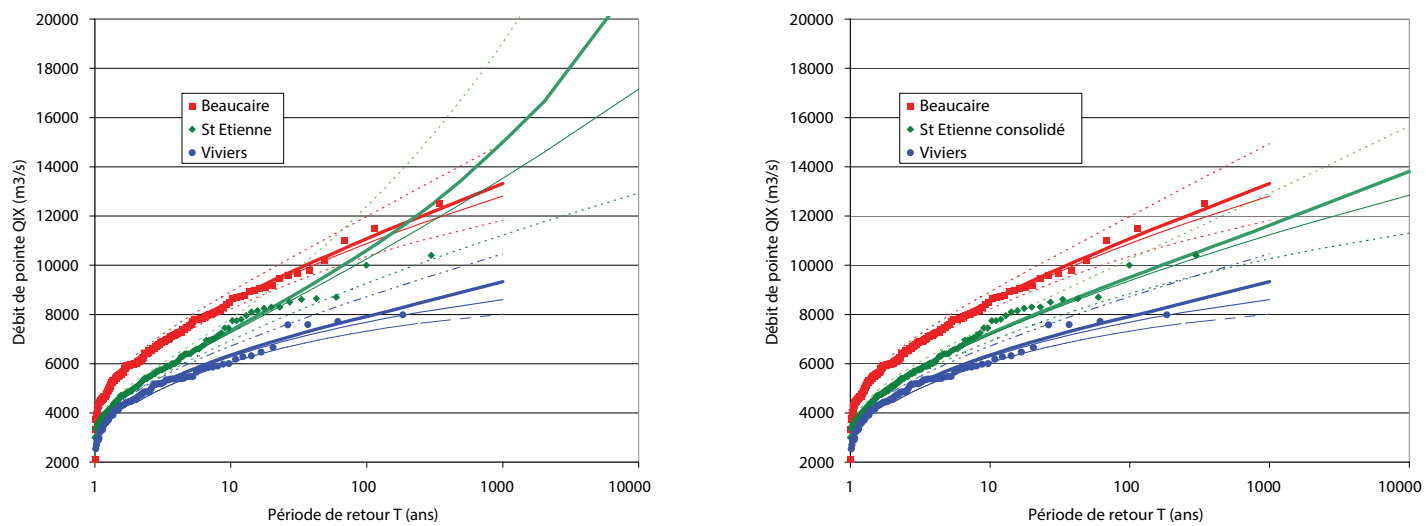

Figure 4 : Ajustement d'une loi GEV sur les débits QIX à Viviers, Saint-Etienne-des-Sorts et Beaucaire : a/ traitement "brut » à Saint-Etienne ; b/ traitement « consolidé amont-aval » à Saint-Etienne.

Le calcul de la distribution du débit de pointe de Saint-Etienne-des-Sorts a donc été réactualisé de la façon suivante. Dans la première version, les paramètres de la loi GEV ont été déterminés par une approche bayésienne, sans donner d'a priori sur la valeur des paramètres de position et d'échelle, et avec un a priori assez large sur la valeur du paramètre de forme $k$. La distribution a priori du paramètre $k$ était une loi normale $N($ moyenne $=0$; écart-type $=0.3)$, soit un intervalle de confiance $\operatorname{IC} 90(k)=[-0.5 ; 0.5]$ qui ne donne aucune préférence sur une des trois lois possibles de la loi GEV (Weibull, Gumbel, Fréchet). Nous avons retenu un nouvel a priori sur le paramètre de forme $k$, qui conditionne l'extrapolation aux crues extrêmes, en prenant une loi normale $N(0.16 ; 0.06)$ qui correspond à l'intervalle de confiance IC90 $(k)$ le plus large entre celui de Viviers $[0.06 ; 0.26]$ et celui de Beaucaire $[-0.01 ; 0.15]$. Il donne la préférence à des valeurs positives du coefficient de forme $k$.

L'estimation des débits de référence à Saint-Etiennedes-Sorts est donnée dans le tableau 1 (valeurs issues de la distribution prédictive ou modale; cf. eq. (3) et (2)). Le nouveau paramètre de forme $k=0.0628$ obtenu $a$ posteriori, en combinant un a priori sur le paramètre $k$ distribué suivant une loi normale $N(0.16 ; 0.06)$, par conditionnement amont-aval avec les informations issues de Viviers et

Tableau 1 : Débits de pointe $\left(\mathrm{m}^{3} / \mathrm{s}\right)$ à Saint-Etienne-des-Sorts à l'aide d'une loi GEV consolidée. Distribution prédictive (modale).

\begin{tabular}{|c|c|c|c|c|c|c|c|}
\hline \multicolumn{4}{|c|}{ Paramètres de la loi GEV consolidée } & \multicolumn{3}{c|}{ Quantiles $Q I X(T)$ en $\mathbf{~ m}^{3} / \mathbf{s}$ pour différentes périodes de retour $T$ (an) } \\
\hline$x_{0}$ & $a$ & $k$ & $I 90 \%(k)$ & $10^{2}$ & $10^{3}$ & $10^{4}$ & $10^{5}$ \\
\hline 4740 & 1160 & 0.0628 & {$[-0.08 ; 0.13]$} & 9510 & 11660 & 13800 & 16100 \\
& & & & $(9370)$ & $(11200)$ & $(12800)$ & $(14200)$ \\
\hline
\end{tabular}


Beaucaire, et la série de Saint-Etienne-des-Sorts (1845-2011), est positif et se situe au niveau de la borne inférieure de l'intervalle choisi a priori. La figure $4 \mathrm{~b}$ montre que cette fois-ci l'ajustement consolidé de la distribution du débit de pointe à Saint-Etienne-des-Sorts est cohérent avec les distributions estimées en amont (Viviers) et en aval (Beaucaire).

Le tableau 2 donne la période de retour associée à chacun des débits issus de la modélisation hydraulique.

Tableau 2 : Périodes de retour des différents débits étudiés au droit de la plateforme industrielle.

\begin{tabular}{|l|c|c|c|c|c|}
\hline Débit de submersion $\left(\mathbf{m}^{3} / \mathbf{s}\right)$ & 14565 & 15929 & 19100 & 22000 & 25711 \\
\hline Période de retour $(\mathbf{a n s})$ & $210^{4}$ & $810^{4}$ & & $>10^{5}$ \\
\hline
\end{tabular}

L'ajustement par une loi GEV a été prolongé pour les besoins de l'étude bien au-delà du domaine classique d'extrapolation d'une distribution des valeurs extrêmes calée sur une série de 150 ans. Habituellement, les quantiles de crue ne sont donnés que jusqu'à la période de retour 1000 ans. On voit ici que le premier débit étudié de $14565 \mathrm{~m}^{3} / \mathrm{s}$ (cote $38.5 \mathrm{~m} \mathrm{NGFO)}$ est supérieur à la crue déca-millénale, et qu'au-delà on dépasse rapidement le niveau des plus fortes crues envisageables estimables. Il faut également noter qu'en l'absence des stations de Viviers et Beaucaire, dotées de séries de données plus robustes, l'analyse hydrologique aurait fourni des débits du Rhône à Saint-Etienne-des-Sorts supérieurs à ceux finalement retenus.

\section{EVALUATION DES INCERTITUDES LIEES À LA MODELISATION HYDRAULIQUE}

Le modèle hydraulique utilisé pour simuler les écoulements des crues du Rhône fait appel à des paramètres qui sont évalués dans une plage de valeurs possibles issues d'un calage sur les observations ou de données bibliographiques. Les questions qui sont posées sont alors les suivantes :

- "Compte tenu des incertitudes relatives à la valeur de chacun des paramètres hydrauliques, quel est l'impact de leur variation sur la cote atteinte au droit de la plateforme industrielle?"

- "Peut-on déterminer un degré de confiance aux valeurs hydrauliques nominales calculées?»

\section{IV.1. Méthodologie}

Ces questions, bien que fondamentales, n'étaient traitées jusqu'alors que par des analyses de sensibilité locale, c'est-à-dire par des simulations complémentaires faisant varier un seul paramètre sur un nombre d'échantillons restreint à quelques unités (de l'ordre de 3 à 5 simulations en général). Grâce aux moyens de calcul contemporains, il est aujourd'hui envisageable de travailler sur de nombreux échantillons.

La démarche qui a été suivie consiste à qualifier les sources d'incertitude du modèle (sur un ensemble de paramètres choisi parmi les plus influents), à quantifier les plages et forme de distribution de ces paramètres variants, puis à les propager afin de disposer de résultats intégrant une quantification de ces incertitudes et clarifiant l'appréciation des marges associées (distribution de la variable d'intérêt).

Il est ici proposé de répondre à ces questions sur la base d'une méthodologie innovante, dont les deux produits sont :

- Analyse de la dispersion des résultats (cote au droit de la plateforme) en fonction de la variation d'un para- mètre hydraulique avec l'appui d'un échantillonnage statistiquement robuste (on parlera d'analyses d'incertitudes mono-paramètre) ;

- Analyse de la dispersion des résultats en fonction de la propagation d'incertitudes (c'est-à-dire la variation de tous les paramètres en même temps).

\section{IV.1.1. Couplage Prométhée-Crue}

Des démarches de propagation d'incertitudes sont mises en œuvre à l'IRSN depuis plusieurs années, notamment sur des codes de calculs de neutronique. Pour cela, l'IRSN a développé l'outil Prométhée ${ }^{1}$ qui facilite le paramétrage et le lancement des codes de calcul et propose des outils statistiques d'interprétation des résultats des calculs. Prométhée est un environnement permettant de lancer un nombre considérable de calculs, dans la démarche de modélisation numérique des incertitudes basée sur une méthode de type Monte Carlo.

Le code de calcul hydrodynamique Crue, développé par $\mathrm{CNR}$, et simulant le comportement hydraulique d'une rivière ou d'un système de canaux, repose sur une intégration numérique des équations de Barré de Saint-Venant appliquées le long de biefs séparés par des profils en travers permettant la représentation physique du tronçon à modéliser. Le code de calcul permet de représenter des réseaux ramifiés et maillés en régime permanent ou transitoire.

Le couplage Prométhée/Crue permet de réaliser des propagations d'incertitudes dans un modèle hydraulique, c'est-à-dire de faire varier plusieurs paramètres d'entrée et de réaliser un nombre important de calculs en parallèle.

\section{IV.1.2. Les paramètres variants (ou variables d'entrée)}

La détermination des paramètres variants, le choix des bornes extrêmes ainsi que le choix des lois de distribution attribuées à ces paramètres est le travail amont à réaliser avant d'entreprendre les calculs de propagation d'incertitudes. Le choix des valeurs minimum et maximum est complexe pour deux raisons :

- Il est nécessaire de réaliser une analyse de sensibilité et non une analyse de scénarios alternatifs : l'encadrement de la valeur nominale doit être « restreint»;

- Le choix des valeurs extrêmes doit être justifié au regard d'éléments d'expertise ou de mesures si elles existent : le coefficient de frottement théorique pourrait être relié à des analyses granulométriques par exemple.

1. Site Internet de l'IRSN, outils scientifiques développés. 
Ces paramètres variants se répartissent en différentes classes : - Paramètres influents fixés par le calage ou paramètre numérique : $[\mathrm{K}]$ coefficient de frottement de Strickler lié à la rugosité des fonds et des bords, [Cs] coefficient de modulation de la débitance des seuils déversants, et [Cd] coefficient de modulation de la débitance du barrage de retenue.

- Conditions aux limites : il est intéressant de disposer d'éléments sur la variation du niveau au droit du site industriel en fonction des variations des conditions hydrologiques ou des configurations des ouvrages : [Qa] débit des affluents, [Qe] forme de l'hydrogramme entrant dans l'aménagement, et [Qus] débit sortant à l'usine.

- Paramètres liés aux scénarios hydrauliques simulés : [Lrupt] largeur de la brèche, [Trupt] écart entre la ligne de charge calculée à un instant $t$ de la crue et la cote de crête du cavalier de l'endiguement, et [Drupt] durée d'établissement d'une brèche.

$\mathrm{Au}$ total, 9 paramètres variants sont donc analysés sur le débit de $14565 \mathrm{~m}^{3} / \mathrm{s}$.

\section{IV.1.3. Lois de distribution des paramètres variants}

Les valeurs issues du calage par l'expert, appelées valeurs nominales par la suite, sont par postulat celles qui ont la plus forte probabilité (valeurs modales). Concernant le choix des lois de distribution, en l'absence d'informations sur les incertitudes, les différentes études antécédentes montrent que l'ensemble des paramètres physiques convergent généralement vers des lois normales.

Considérant que l'ensemble des valeurs possibles des paramètres sont comprises dans ces gammes de variation, on associe les bornes de la gamme de variation aux bornes d'intervalle de confiance à $95 \%$ du paramètre. On peut en déduire l'écart type qui sera utilisé pour les lois normales (intervalle de confiance à $95 \%=2 \sigma$ ).

Le tableau 3 présente les paramètres variants retenus dans le cadre de cette étude.

\section{IV.1.4. Echantillonnage selon la méthode de Monte-Carlo (tir statistique)}

La méthode de Monte-Carlo permet la simulation de tirages aléatoires des variables d'entrée des modèles, sur la base de distributions statistiques. L'expression "simulation de Monte-Carlo » recouvre une série de techniques destinées à résoudre des problèmes complexes toujours probabilistes par l'introduction d'échantillonnages aléatoires.

Le nombre de tirs ici réalisé est de 300 afin d'assurer la convergence des résultats et le traitement des calculs dans Prométhée. Un calcul est par conséquent l'addition de 300 simulations (avec 1 ou $n$ paramètres variants). Un test statistique d'égalité des variances a été réalisé avec un calcul contenant 1000 échantillons comparé au même calcul à 300 échantillons. Le calcul de la valeur montre que l'hypothèse nulle n'est pas rejetée, validant le nombre d'échantillon pris en compte dans l'ensemble des calculs : les résultats sont identiques entre 300 et 1000 échantillons avec un intervalle de confiance de $95 \%$.

\section{IV.1.5. La variable d'intérêt (ou variable de sortie)}

La variable d'intérêt choisie est la cote définie au droit de la plateforme industrielle. Les grandeurs calculées et analysées sont donc des couples de valeurs du type $\{$ COTE ; paramètre(s) variant(s)\}. Elles permettent de caractériser le comportement du " système hydraulique » étudié.

Sur l'histogramme de la figure 5 sont indiquées en vert les bornes de l'intervalle de confiance à $95 \%$ autour de la moyenne, et en rouge la valeur nominale. Ceci permet d'estimer si la correspondance (ou non-correspondance) de la valeur nominale et de la moyenne de l'échantillon est statistiquement significative.

\section{IV.2. Enseignements issus de l'application au système hydraulique environnant le site industriel}

S'agissant de la première application opérationnelle hydraulique d'envergure pour l'outil Prométhée à la CNR, le fonctionnement et les temps de traitement associés ont été jugés très performants vis-à-vis des 32 kilomètres de fleuve modélisés et de la complexité des organes hydrauliques 1D composant ce linéaire, qui plus est pour la représentation de phénomènes extrêmes avec contribution forte des lits majeurs. Pour information, environ 7000 simulations ont été nécessaires pour la réalisation de cette étude de sensibilité aux paramètres hydrauliques (temps de calcul moyen de quatre heures par calcul).

\section{IV.2.1. Résultats de propagation mono-paramètre}

Globalement, selon les essais de propagation mono-paramètre, les résultats d'un tir statistique à partir d'une distribution centrée en entrée produisent une distribution également centrée en sortie (réponse linéaire du modèle hydraulique). Un certain nombre de paramètres réagissent conformément à l'intuition hydraulique : [Cd], [Qus], [Cs] et [Qa]. De plus, la méthode apporte des éléments quantitatifs inédits sur l'amplitude de dispersion pour chacun des paramètres, ainsi qu'une hiérarchisation sans dépendance entre ces paramètres.

Tableau 3 : Récapitulatif des paramètres variants et bornes extrêmes.

\begin{tabular}{|c|l|l|l|l|}
\hline Symbole & \multicolumn{1}{|c|}{ Description } & \multicolumn{1}{|c|}{ Valeur min. } & Valeur nominale & \multicolumn{1}{c|}{ Valeur max. } \\
\hline$[\mathbf{Q a}]$ & Coefficient de modulation du débit des affluents & $0.5(-50 \%)$ & 1 & $1.5(+50 \%)$ \\
\hline$[\mathbf{Q e}]$ & Coefficient de modulation de la forme Q(t) & crue Ardèche & crue Mixte & crue Rhône \\
\hline$[Q \mathbf{Q u s}]$ & Débit sortant à l'usine de Caderousse & $1500 \mathrm{~m}^{3} / \mathrm{s}$ & $1650 \mathrm{~m}^{3} / \mathrm{s}$ & $1800 \mathrm{~m}^{3} / \mathrm{s}$ \\
\hline$[\mathbf{C s}]$ & Coefficient de modulation de la débitance des seuils & $0.81(-10 \%)$ & 0.9 & $0.99(+10 \%)$ \\
\hline$[\mathbf{C d}]$ & Coefficient équivalent de débit de Marchi du barrage & $1.12(-10 \%)$ & 1.18 & $1.24(+10 \%)$ \\
\hline$[\mathbf{K}]$ & Coefficient de modulation des coefficients de Strickler & $0.9(-10 \%)$ & 1 & $1.1(+10 \%)$ \\
\hline$[$ Lrupt] & Largeur de la brèche & $50 \mathrm{~m}$ & $100 \mathrm{~m}$ & $150 \mathrm{~m}$ \\
\hline$[$ Drupt $]$ & Durée de la rupture & $0.5 \mathrm{~h}$ & $1 \mathrm{~h}$ & $1.5 \mathrm{~h}$ \\
\hline$[$ Trupt $]$ & Date T de la rupture & $-4 \mathrm{~h}$ & $\mathrm{~T} 0$ & $+11 \mathrm{~h}$ \\
\hline
\end{tabular}




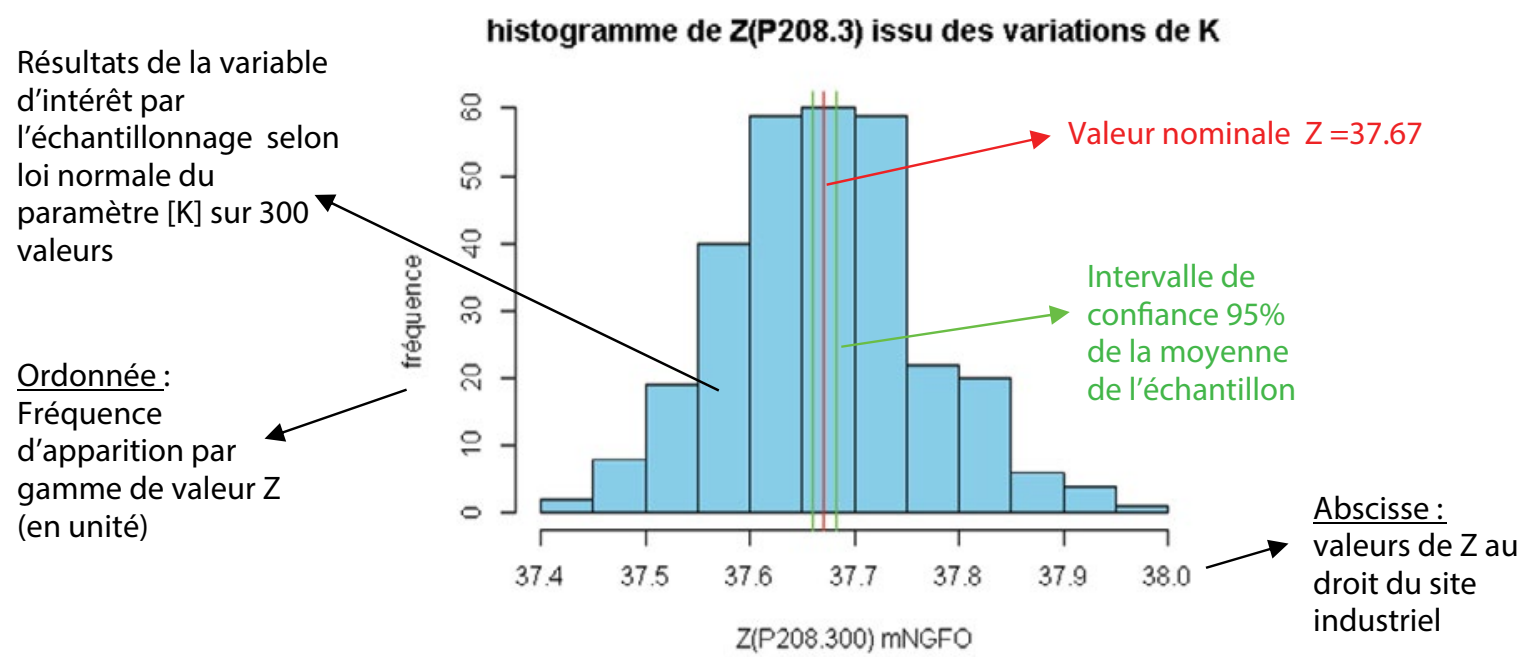

Figure 5 : Distribution de la variable d'intérêt $Z$ selon échantillonnage du paramètre $K$ selon la loi normale.

Les principaux éléments propres à la démarche stochastique lors de l'analyse de propagation mono-paramètre sont les suivants :

- Le paramètre le plus influant sur la cote au droit du site industriel est le coefficient de Strickler quel que soit le scénario étudié. Sur la base de la distribution proposée, l'amplitude estimée est de 35 à $55 \mathrm{~cm}$ (respectivement scénarios 1 et 2 sans et avec rupture des endiguements). Le niveau zéro de la plateforme n'est pas atteint. Les conditions hydrauliques dans le Rhône au droit du site étant favorables à l'établissement de dunes, des tests alternatifs à $[\mathrm{K}]-12$ points ont été également réalisés sans atteindre la cote critique, avec mise en lumière d'effet non-linéaire.

- La largeur de brèche [Lrupt] possède un niveau d'influence comparable au coefficient précédent : l'effet est non linéaire traduisant une quasi non-sensibilité à une largeur de brèche supérieure à la valeur nominale (figure 6). Dans une moindre mesure, l'incertitude sur le moment d'activation de la brèche produit une amplitude de $32 \mathrm{~cm}$ mais uniquement en cas de brèche tardive (forte non-linéarité).

\section{IV.2.2. Résultats de propagation multi-paramètres}

La propagation d'incertitudes en modélisation hydraulique ouvre probablement une nouvelle ère dans l'utilisation des codes numériques 1D: la démarche aléatoire apporte

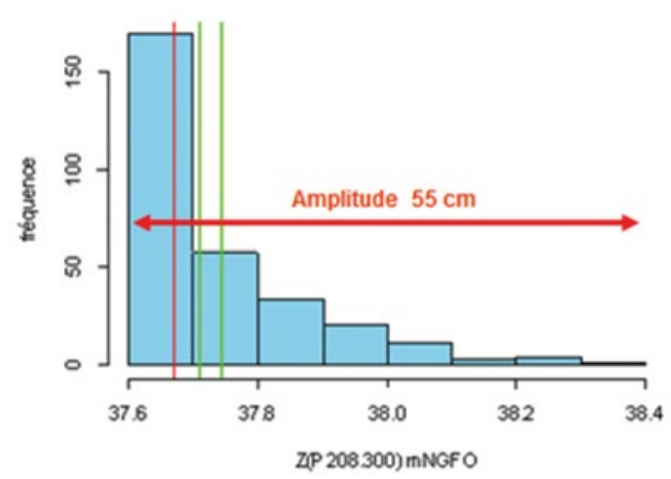

Figure 6 : Histogramme de propagation du paramètre [Lrupt]. des éléments quant à l'importance et la hiérarchisation des différents paramètres. Dans le cas d'application à ce site industriel, la propagation simultanée des incertitudes des paramètres $[\mathrm{K}]$ et [Lrupt] induit une dispersion très forte et non centrée des résultats du scénario 2 (avec rupture) au droit du site: $1,37 \mathrm{~m}$ pour l'analyse avec $[\mathrm{K}]+/-10 \%$. La propagation d'incertitudes dans ce scénario avec rupture induit une amplitude trois fois plus élevée qu'une propagation similaire dans le scénario 1 (sans rupture). Ceci s'explique d'une part par l'absence de la compensation hydraulique des déversements sur les endiguements de la retenue et d'autre part par le cumul d'incertitudes fortes liées à la rupture de l'endiguement (largeur). Le paramètre $[\mathrm{K}]$ est le fort contributeur de la dispersion.

D'autre part, le calcul prouve que la somme de toutes les incertitudes des différents paramètres induit une dispersion non cumulative par rapport à la simple analyse faite par paramètres indépendants (effet des covariances). Cela illustre l'intérêt d'une approche par propagation par rapport à une étude nominale, même avec des valeurs de paramètres pessimistes.

Il faut notamment retenir que ce type de calcul aléatoire permet de réaliser des analyses de risques de concomitance: c'est le cas par exemple, figure 7 représentant un scénario alternatif, de la combinaison de paramètres légèrement défavorables du [Cd], [Qus] et [K] du scénario 1 (hypothèse de non rupture des endiguements) qui engendre des valeurs extrêmes au droit du site industriel (au-dessus du niveau zéro de $38,70 \mathrm{~m}$ NGFO).

\section{IV.2.3. Perspectives}

Rappelons les axes de progression suivants de cette nouvelle méthode d'appréhension de l'impact des incertitudes en modélisation hydraulique : réflexion sur d'éventuelles méthodes déterministes d'évaluation des bornes extrêmes des paramètres variants, expérimentations à partir de lois de distribution différentes qui impactent nécessairement l'analyse statistique à produire, optimisation des analyses statistiques : il est à noter les fortes potentialités de l'outil Prométhée développé par l'IRSN qui propose des méthodes probabilistes (méthodes FAST et MORRIS). Celles-ci étudient la variabilité des sorties relativement à la variabilité des entrées. 

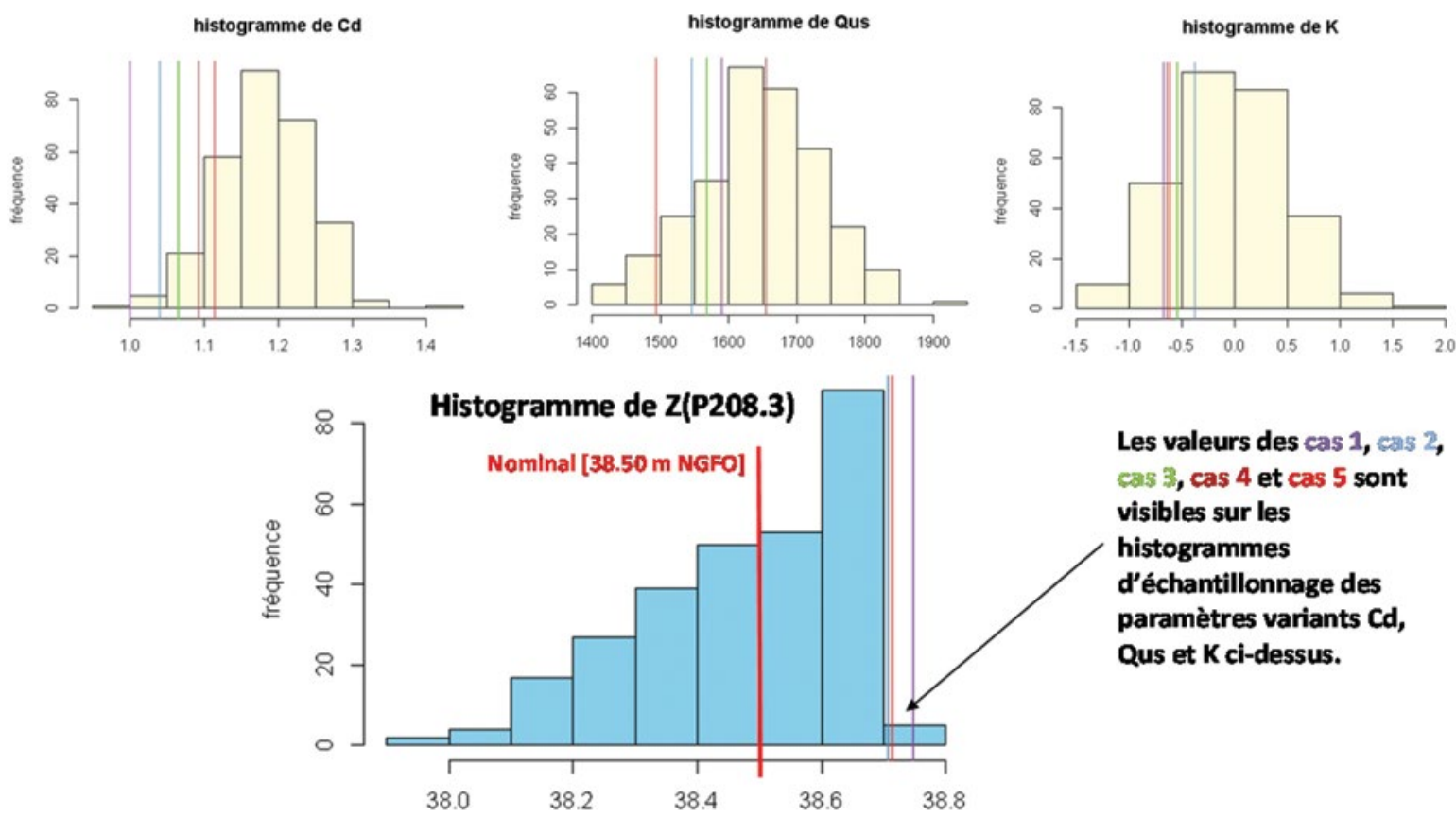

Figure 7 : Distribution des paramètres variants (jaune) et distribution de sortie de la variable d'intérêt (bleu) par propagation multi-paramètres.

\section{CONCLUSIONS}

La présente étude a permis de répondre à la question de l'exploitant sur le débit de submersion de la plateforme industrielle. En l'état actuel des connaissances, seul un scénario quasi improbable de non rupture des endiguements CNR sous une charge hydraulique forte et continue conduirait à une possibilité de submersion continue, pour un débit estimé à près de $16000 \mathrm{~m}^{3} / \mathrm{s}$, avec une probabilité annuelle de submersion de l'ordre de $10^{-5}$. On se situe ici en limite du pouvoir d'estimation des débits extrêmes avec de fortes incertitudes liées aux choix de modélisation statistique. Les résultats obtenus dans tous les scénarios montrent un plafonnement des cotes de submersion. Dans la gamme de ces débits extrêmes, chaque $\mathrm{cm}$ de cote de ligne d'eau correspond à une augmentation très importante de l'aléa.

Plusieurs points sur l'étude des incertitudes nous amènent à une série de recommandations :

- Un premier point concerne les modalités d'intégration des incertitudes hydrologiques. Plutôt que de raisonner sur la borne supérieure d'un intervalle de confiance, avec un choix arbitraire de sa largeur et une forte sensibilité au choix de la distribution (Gumbel ou GEV), il nous semble que l'emploi d'une distribution prédictive permet de progresser dans les modalités de prise en compte des incertitudes. Il reste toutefois que des questions subsistent sur la cohérence des données de débit anciennes, entre débit de pointe et débit journalier, et de l'amont vers l'aval. Un travail complémentaire d'analyse des séries historiques du Rhône sur les derniers siècles permettrait de conforter les estimations de crue extrême, et également de positionner la crue de 1856 au-delà de la période 1845-2011.

- Enfin, l'analyse de sensibilité sur la propagation des incertitudes sur le modèle hydraulique a permis de mettre en évidence les sources d'erreur les plus importantes : outre l'hypothèse de départ sur le caractère résistant ou non à la submersion des endiguements CNR qui change radicalement les conclusions (les écrêtements du débit consécutif à l'activation rapide du champ d'inondation rive gauche, par rupture de digue, limitent la cote de la ligne d'eau à un niveau inférieur à celui de la plateforme industrielle), le paramètre le plus sensible est le paramètre de Strickler. A noter les fortes potentialités de l'outil statistique Prométhée développé par l'IRSN qui permet de propager les incertitudes simultanées de plusieurs paramètres hydrauliques et conduit à une estimation plus réaliste des incertitudes que la simple sommation des incertitudes sur chaque paramètre.

\section{REFERENCES}

ASN (2013) - Protection des installations nucléaires de base contre les inondations externes. Guide, version du 08/01/2013. 13

Centre D’etude De L'equipement Mediterranee (2001) Recensement et quantification des sources d'incertitudes externes. Étude de sensibilité des modélisations 1D. 67 pages

Compagnie Nationale Du Rhone (2005) - CRUE9 logiciel de modélisation filaire des écoulements à surface libre

Fahsi A., Soulaïmani A. Eт Tchamen G.W. (2011) — Propagation des incertitudes en modélisation hydraulique des rivières. $\mathrm{La}$ Houille Blanche. 6 : 42-48

Fisher R. A., Et L. H. C. Tippett (1928) — Limiting forms of the frequency distribution of the largest or smallest member of a sample. Mathematical Proceedings of the Cambridge Philosophical Society. 24(02) : 180-190

Garçon R., Bossard E., Lang M. Et Le Clerc S. (2002) Revisiter la notion de scénario hydrologique de référence pour la caractérisation du risque d'inondation. Rapport commun EDF/DTG Cemagref pour le compte du Ministère de l'Écologie et du Développement Durable, Programme Risque Inondation RIO $1.76 \mathrm{p}$

Sauquet E., Ramos M.H., Chapel L. Et Bernardara P. (2008) Streamflow scaling properties:investigating characteristic scales from different statistical approaches., doi: 10.1002/hyp.6952 (erratum: doi: 10.1002/hyp.7192). Hydrological Processes. 22(17) : 3462-3475 